\title{
Reconstruction and analysis of competitive endogenous RNA network reveals regulatory role of long non-coding RNAs in hepatic fibrosis
}

\author{
HUI JIANG ${ }^{1,2^{*}}$, FURONG WU $^{3^{*}}$, NANNAN JIANG ${ }^{2}$, JIARONG GAO $^{2}$ and JIAFU ZHANG \\ ${ }^{1}$ Experimental Center of Clinical Research; ${ }^{2}$ Department of Pharmacy, \\ The First Affiliated Hospital of Anhui University of Chinese Medicine, Hefei, Anhui 230031; \\ ${ }^{3}$ Department of Pharmacy, Anhui Provincial Hospital, Hefei, Anhui 230001, P.R. China
}

Received February 24, 2019; Accepted August 12, 2019

DOI: $10.3892 / \mathrm{mmr} .2019 .10682$

\begin{abstract}
Hepatic fibrosis (HF), one of the leading global health problems, is defined as aberrant and excess production of extracellular matrix components. The pathogenesis of HF is complex and poorly understood. Long non-coding RNAs (LncRNAs) can interact with microRNAs (miRNAs) as competing endogenous RNAs (ceRNAs) to regulate the expression of target genes, which play a significant role in the initiation and progression of HF. In the present study, the LncRNA-associated ceRNA network was reconstructed based on LncRNA, miRNA and mRNA expression profiles that were downloaded from National Center for Biotechnology Information Gene Expression Omnibus. Bioinformatics assessments including Gene Ontology (GO) and Kyoto Encyclopedia of Genes and Genomes pathway analyses were performed with Database for Annotation, Visualization and Integration Discovery. The ceRNA network was composed of 220 LncRNA nodes, 24 miRNA nodes, 164 mRNA nodes and 1,149 edges. Functional assays identified that a total of $338 \mathrm{GO}$ terms and 25 pathways, including regulation of cytokine and collagen, and the transforming growth factor- $\beta$ and Toll-like receptor signaling pathways, were significantly enriched. In addition, 4 LncRNAs (NONMMUT036242, XR_877072, XR_378619 and XR_378418) were highly related to HF and thereby chosen as key LncRNAs. The present study uncovered a ceRNA network that could further the understanding of the mechanisms underlying HF development and provide potential novel markers for clinical diagnosis and targets for treatment.
\end{abstract}

Correspondence to: Dr Jiafu Zhang, Department of Pharmacy, The First Affiliated Hospital of Anhui University of Chinese Medicine, 117 Meishan Road, Hefei, Anhui 230031, P.R. China E-mail: zyfyzjf@163.com

${ }^{*}$ Contributed equally

Key words: hepatic fibrosis, long non-coding RNAs, competitive endogenous RNA network

\section{Introduction}

Hepatic fibrosis (HF), a reversible pathophysiological process, is characterized by an increased and altered deposition of extracellular matrix (ECM) proteins that results in excessive tissue scarring and promotes chronic liver injury $(1,2)$. Due to its end-stage outcome (cirrhosis), it is responsible for high morbidity and mortality (3). Over the past several years, great efforts have been made to provide insight into the molecular mechanisms underlying HF $(4,5)$. However, the precise molecular mechanisms still remain to be determined (6). Therefore, elucidating the pathogenesis of liver fibrosis and identifying novel treatment strategies, including potential biomarkers and therapeutic targets to combat $\mathrm{HF}$, are of great importance.

Accumulating evidence suggests that although they have no or little protein-coding capacity, various non-coding RNAs (ncRNAs) serve as master regulators that affect expression levels of dozens or even hundreds of target genes $(7,8)$. Long (L)ncRNAs are defined as RNA molecules that are $>200$ nucleotides in length, transcribed by RNA polymerase II and lacking an open reading frame (9). LncRNAs have been found to be associated with the pathogenesis of different types of diseases by regulating diverse biological processes, such as transcription, splicing and translation (10-13). To date, >50,000 LncRNAs have been cloned and identified in the human genome; however, only a small number of these have been functionally annotated (14). Improved knowledge has suggested that LncRNAs can regulate microRNA (miRNA) abundance by binding and sequestering them, thereby regulating the expression of target mRNAs (15). Thus, it has been demonstrated that an efficient way to the infer potential function of LncRNAs is by studying associated miRNAs and/or mRNAs whose functions have been annotated. This association has been demonstrated in several diseases, but has not been studied in HF (16-18).

The present study reconstructed a competitive endogenous RNA (ceRNA) network using the data from National Center for Biotechnology Information (NCBI; https://www.ncbi.nlm. nih.gov/) Gene Expression Omnibus (GEO; https://www.ncbi. nlm.nih.gov/geo/), based on the ceRNA theory, a new RNA language that mRNAs, transcribed pseudogenes, and LncRNAs 'talk' to each other using miRNA response elements (19). 
Subsequently, Gene Ontology (GO; http://geneontology.org/) and Kyoto Encyclopedia of Genes and Genomes (KEGG; https://www.kegg.jp/) pathway analysis displayed that a number of processes overrepresented in $\mathrm{HF}$ were related to the regulation of cytokine and collagen, and the transforming growth factor- $\beta$ (TGF- $\beta$ ) and Toll-like receptor (TLR) signaling pathways. Furthermore, the ceRNA network analysis identified that 4 LncRNAs (NONMMUT036242, XR_877072, XR_378619 and XR_378418) were highly related to HF, suggesting that these LncRNAs were key LncRNAs, and could be used as potential diagnosis markers and treatment targets. Thus, the present study may help expand the understanding of the possible mechanism of HF from the perspective of LncRNAs, and provide some new potential LncRNA markers for the clinical diagnosis and treatment of HF.

\section{Materials and methods}

Raw data. Mouse miRNA expression data were downloaded from NCBI GEO (GSE77271) (20), based on the SurePrint G3 Mouse miRNA Microarray. A total of 6 samples were included in the dataset: 3 samples were the control group and 3 samples were the liver fibrosis group, which was induced using carbon-tetrachloride $\left(\mathrm{CCl}_{4}\right)$. Mouse LncRNA and mRNA expression data were also downloaded from NCBI GEO (GSE80601) (21), generated using an Affymetrix GeneChip Mouse Exon 1.0 ST Array. A total of 10 samples were included in this dataset: 5 samples were the control group and 5 samples were the liver fibrosis group, which was also induced by $\mathrm{CCl}_{4}$.

Gene chip probe re-annotation. Based on the LncRNA classification pipeline constructed in our previous study (22), a number of LncRNAs represented on the Affymetrix microarrays were identified. First, the latest version of the NetAff $\mathrm{x}^{\mathrm{TM}}$ Annotation File (MoEx-1_0-st-v1 Probeset Annotations, CSV Format, release 36; uploaded 7/6/16) was obtained from the Affymetrix official website (http://www.affymetrix.com/support/technical/byproduct.aff $x$ ?product=moexon-st). This annotation file was mapped to the MoEx-1_0-st-v1 probe sets ID. Second, the probe sets were annotated in the Refseq database (https://www. ncbi.nlm.nih.gov/refseq/); those IDs beginning with 'NR' were retained, and transcript IDs labeled with 'NP' were deleted. Probe sets from the Ensembl database (https://www. ensembl.org/index.html) and the online software BioMart v95 (https://www.ensembl.org/info/data/biomart/index.html) were applied to convert Affymetrix microarray IDs to Ensembl IDs, together with the corresponding gene type, and only RNAs type which were annotated as 'lincRNA', 'sense_intronic', 'processed_transcript', 'antisense', 'sense_overlapping', '3prime_overlapping_ncrna' or 'misc_RNA' were retained. The probe sets that annotated in Noncode 44 (http:// www. noncode.org/) were all retained. Next, based on the above two steps, probe set IDs annotated as 'microRNA', 'snoRNAs', 'pseudogenes' and other small RNAs were removed.

Screening of differentially expressed LncRNAs, miRNAs and mRNAs. First, differential probes of GSE77271 and GSE80601 were selected with thresholds of fold change (FC) $>1.5$ and $\mathrm{P}<0.05$ using GEO2R (https://www.ncbi.nlm.nih. gov/geo/geo $2 \mathrm{r} /$ ). Second, differential probes were matched to miRNAs in GSE77271, and LncRNAs and mRNAs in GSE80601. Then, the differentially expressed LncRNAs (DELs), miRNAs (DEMis) and mRNAs (DEMs) were identified.

ReconstructionceRNAnetwork. The LncRNA-miRNA-mRNA network was reconstructed based on ceRNA theory as described previously (7). DEL sequence were obtained from Refseq, Noncode and Ensemble databases, whereas DEMi sequences were obtained from miRBase (http://www.mirbase. org/). Then, the target LncRNAs of miRNAs were predicted by MiRanda (http://www.microrna.org, release 2010) (23). The target mRNAs of miRNAs were predicted by MiRanda and TargetScan v7.2 (http://www.targetscan.org) (24). mRNAs and LncRNAs that were targeted and negatively co-expressed with a certain common miRNA were identified as LncRNA-miRNA-mRNA co-expression competing triplets. The ceRNA network was reconstructed by assembling all co-expression competing triplets, and was visualized using Cytoscape v3.2.8 (25) software. Simultaneously, all node degrees of the LncRNA-miRNA-mRNA network were calculated.

Functional enrichment analysis. To assess functional enrichment, GO $(26,27)$ and KEGG pathway $(28,29)$ analyses of mRNAs in the ceRNA network were performed using Database for Annotation, Visualization, and Integration Discovery (v6.8; https://david.ncifcrf.gov/). Then, a false discovery rate (FDR) was calculated to correct the P-value, and FDR $<0.05$ was selected as the threshold.

Reconstruction of the key LncRNA-miRNA-mRNA sub-networks. In order to evaluate the key LncRNAs, several topological properties, such as the hub nodes and relationship pairs, were considered. First, the hub nodes of LncRNAs with node degree $>5$ were selected. Second, the first relationship pairs of LncRNA-miRNA and the secondary relationship pairs of miRNA-mRNA were calculated. The LncRNAs whose total relationship pairs were in the top 4 were selected as key LncRNAs. Then, key LncRNA-miRNA-mRNA sub-networks were visualized using Cytoscape software. For further analysis, GO and KEGG pathway annotations for each of the key LncRNAs were analyzed by using their original mRNA neighbors in the key LncRNA-miRNA-mRNA sub-networks.

\section{Results}

Identification of DELs, DEMis and DEMs. Based on the cut-off criteria, a total of 254 DELs (107 significantly up- and 147 downregulated), 25 DEMis (12 significantly up- and 13 downregulated) and 472 DEMs (308 significantly up- and 164 downregulated) in the GSE77271 and GSE80601 dataset were identified.

LncRNA-miRNA-mRNA network. To further understand how LncRNAs regulate mRNAs through interactions with miRNAs in HF, a ceRNA network was reconstructed based on the above data and visualized. As presented in Fig. 1, the ceRNA network was composed of 220 LncRNA nodes, 24 miRNA nodes, 164 mRNA nodes and 1,149 edges. Base on 


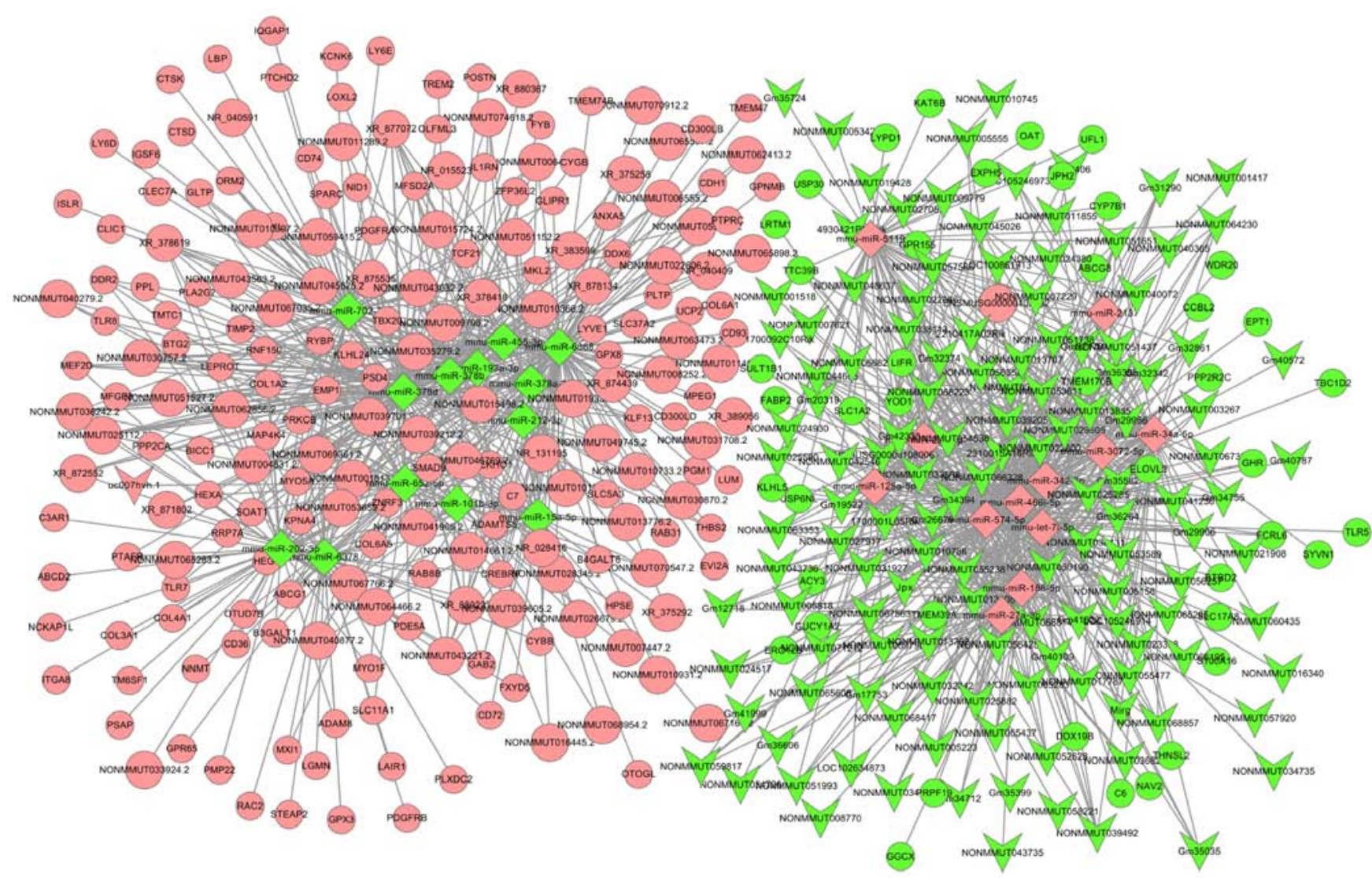

Figure 1. LncRNA-miRNA-mRNA network. The arrowheads represent LncRNAs, rhombuses represent miRNAs, circles represent mRNAs; upregulated genes are in pink, downregulated genes are in green. LncRNA, long non-coding RNA; miRNAs microRNAs.

the LncRNA-miRNA-mRNA network, it was found that one LncRNA can interact with multiple miRNAs and mRNAs, one miRNA can interact with multiple LncRNAs and mRNAs, and one mRNA can interact with multiple LncRNAs and miRNAs; this indicated that the expression profiles of LncRNAs, miRNAs and mRNAs were significantly associated.

Functional enrichment analysis of LncRNAs based on the ceRNA network. To explore the biological functions of LncRNAs during the development of HF, GO and KEGG analyses were conducted. GO analysis revealed that a total of $338 \mathrm{GO}$ terms, including platelet-derived growth factor binding, and regulation of cytokine and collagen, were significantly altered. The top 30 significantly GO terms are shown in Fig. 2A. KEGG pathway analysis indicated that 25 pathways were significantly enriched, including the TGF- $\beta$, TLR and peroxisome proliferator-activated receptor signaling pathways. The top 30 pathways were demonstrated in Fig. 2B.

Topological analysis of the ceRNA network. In general, a higher degree indicates that a node in the ceRNA network was a hub that participated in more ceRNA interactions. According to a previous study by Han et al (30), in which they defined a hub as a node degree $>5$, the present study found that 77 nodes could be chosen as hub nodes, including 43 LncRNAs, 24 miRNAs and 10 mRNAs. The results are shown in Fig. 3. In addition, the number of first relationship LncRNA-miRNA pairs and secondary relationship miRNA-mRNA pairs of hubs were calculated. The results were demonstrated in Table I. On the basis of total relationship pairs, 4 LncRNAs (NONMMUT036242, XR_877072, XR_378619 and XR_378418) were selected as the key LncRNAs.

Key LncRNA-miRNA-mRNA sub-networks. The new key LncRNA-mRNA sub-networks were reconstructed by extracting the key LncRNAs and their linked miRNAs and mRNAs from the triple global network. As presented in Fig. 4, the LncRNA NONMMUT036242-miRNA-mRNA sub-network was composed of 8 miRNA nodes, 112 mRNA nodes and 211 edges. The LncRNA XR_877072-miRNA-mRNA sub-network was composed of 10 miRNA nodes, 120 mRNA nodes and 220 edges. The LncRNA XR_378619-miRNA-mRNA sub-network was composed of 7 miRNA nodes, 109 mRNA nodes and 197 edges. The LncRNA XR_378418-miRNA-mRNA sub-network was composed of 7 miRNA nodes, 100 mRNA nodes and 178 edges.

Functional prediction of key LncRNAs-miRNA-mRNA sub-networks. GO and KEGG pathway annotations were further performed for the 4 key LncRNAs. The results revealed that $335 \mathrm{GO}$ terms and 21 pathways were significantly enriched in the LncRNA NONMMUT036242-miRNA-mRNA sub-network, 230 GO terms and 23 pathways were significantly enriched in the LncRNA XR_877072-miRNA-mRNA sub-network, 399 GO terms and 27 pathways were significantly enriched in the LncRNA XR_378619-miRNA-mRNA sub-network, 200 
A

Top 30 of GO enrichment

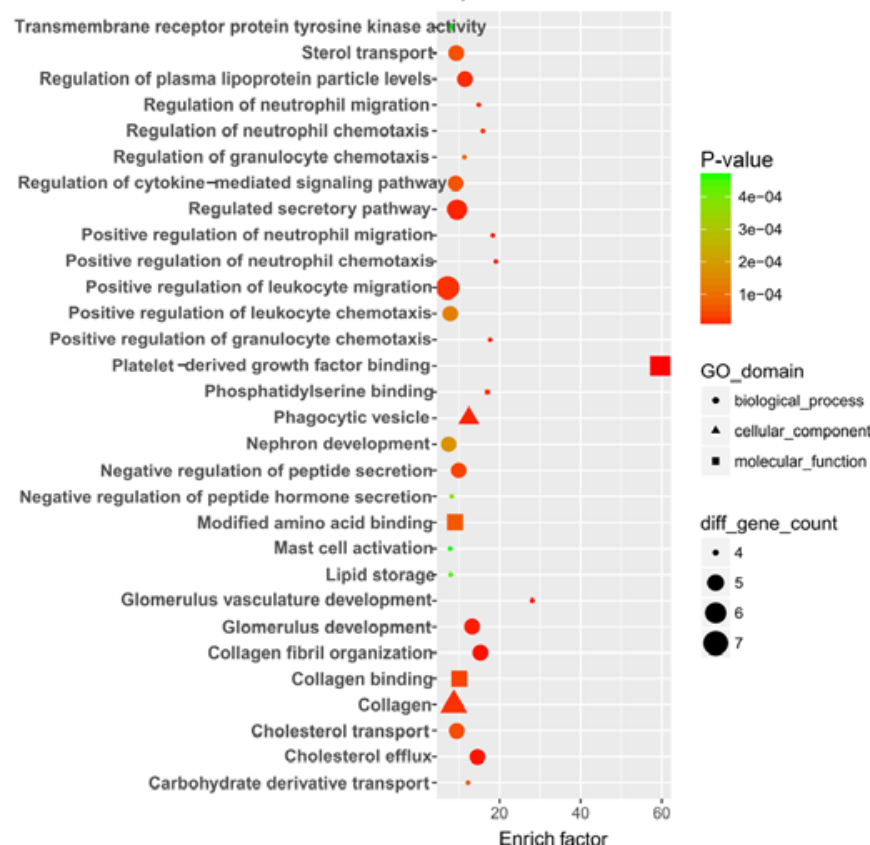

B

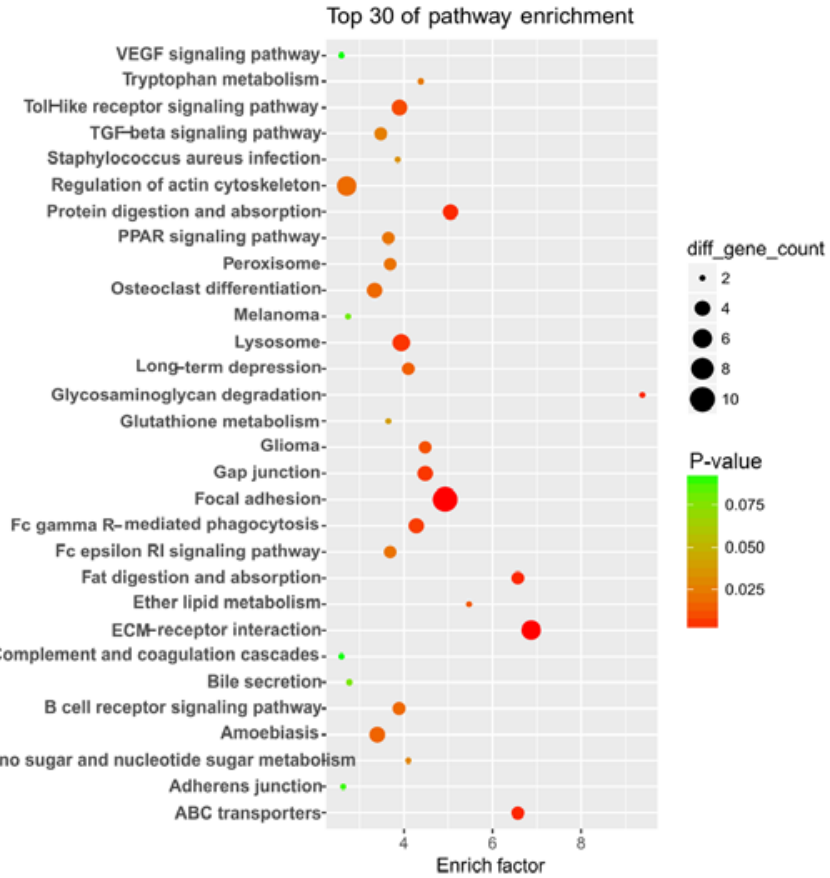

Figure 2. GO and KEGG pathway analysis of differentially expressed mRNAs. (A) Top 30 enriched GO terms. (B) Top 30 changes in KEGG pathway enrichment. Circles represent biological process, triangles represent cellular component and squares represent molecular function. The different colors from green to red represent the P-value. The deeper the color, the more significant the P-value. The different sizes of the shapes represent the gene count number. GO, Gene Ontology; KEGG, Kyoto Encyclopedia of Genes and Genomes.
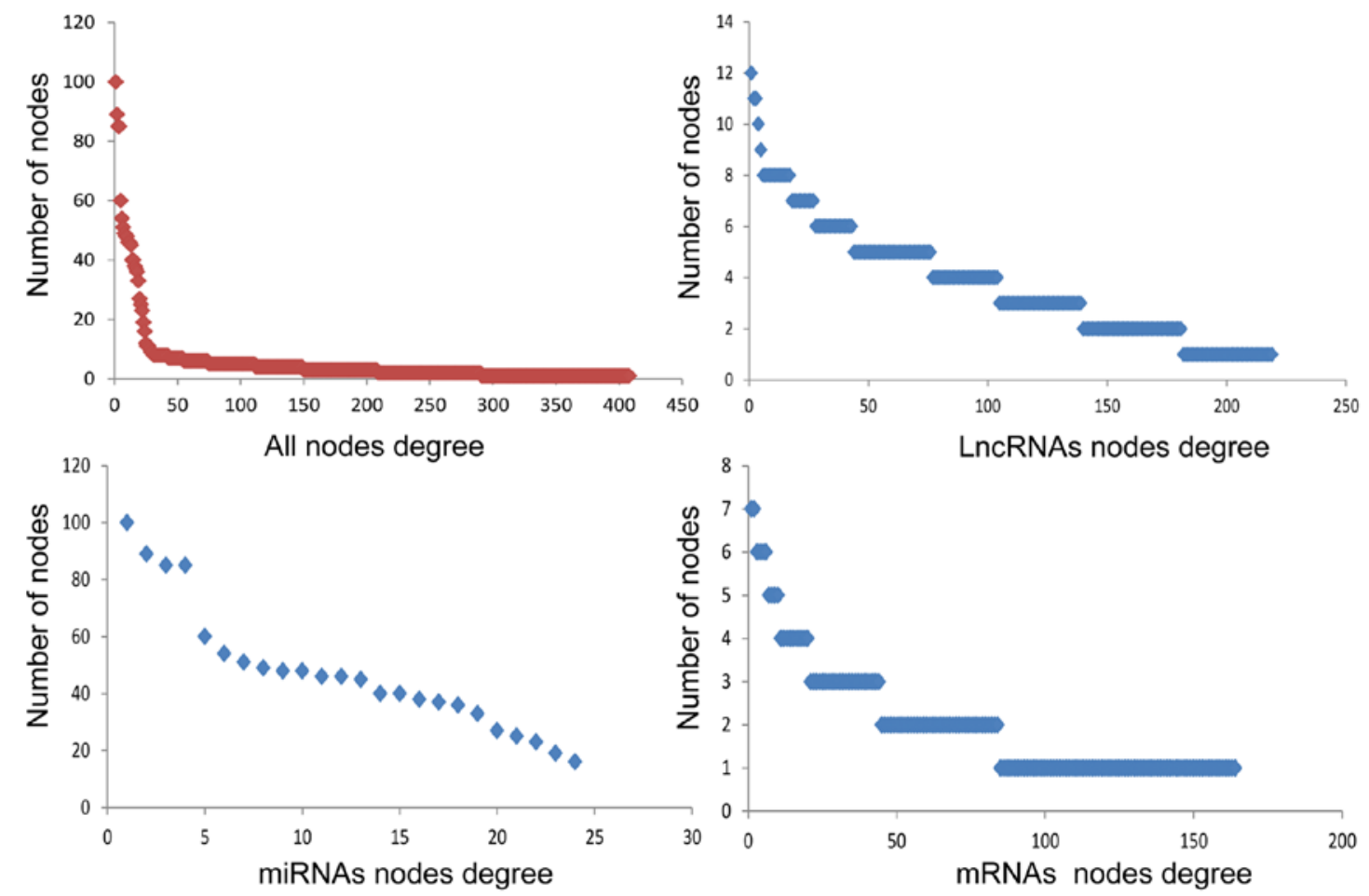

Figure 3. Node degree analysis of the LncRNA-miRNA-mRNA network. LncRNA, long non-coding RNA; miRNAs microRNAs.

GO terms and 18 pathways were significantly enriched in the LncRNA XR_378418-miRNA-mRNA sub-network. The top 30 significant GO terms are shown in Fig. 5 and the top 30 enriched pathways shown in Fig. 6. It is notable that some common GO terms such as 'regulation of cytokine-mediated signaling pathway', 'regulation of plasma lipoprotein particle levels' and 'collagen', and some common pathways such as 'Wnt signaling pathway, 'TGF- $\beta$ signaling pathway' and 'Toll-like receptor signaling pathway' were enriched for all 4 sub-networks. 
Table I. LncRNA-miRNA and miRNA-mRNA pairs of network hubs.

\begin{tabular}{|c|c|c|c|c|}
\hline Number & Gene name & LncRNA-miRNA pairs & miRNA-mRNA pairs & Total number \\
\hline 1 & NONMMUT036242 & 8 & 203 & 211 \\
\hline 2 & XR_877072 & 10 & 199 & 209 \\
\hline 3 & XR_378619 & 7 & 187 & 194 \\
\hline 4 & XR_378418 & 7 & 171 & 178 \\
\hline 5 & NONMMUT025112 & 8 & 161 & 169 \\
\hline 6 & NONMMUT069361 & 8 & 159 & 167 \\
\hline 7 & NONMMUT041965 & 7 & 152 & 159 \\
\hline 8 & NONMMUT039701 & 7 & 144 & 151 \\
\hline 9 & NONMMUT067035 & 7 & 133 & 140 \\
\hline 10 & NONMMUT030757 & 6 & 127 & 133 \\
\hline 11 & NONMMUT043221 & 6 & 113 & 119 \\
\hline 12 & NONMMUT010366 & 6 & 106 & 112 \\
\hline 13 & NONMMUT053659 & 6 & 87 & 93 \\
\hline 14 & Gm34394 & 12 & 77 & 89 \\
\hline 15 & Gm41622 & 11 & 77 & 88 \\
\hline 16 & Gm35562 & 11 & 77 & 88 \\
\hline 17 & NONMMUT022066 & 8 & 74 & 82 \\
\hline 18 & Gm29966 & 8 & 74 & 82 \\
\hline 19 & NONMMUT013707 & 8 & 72 & 80 \\
\hline 20 & NONMMUT051651 & 7 & 72 & 79 \\
\hline 21 & NONMMUT058428 & 7 & 66 & 73 \\
\hline 22 & NONMMUT033508 & 8 & 64 & 72 \\
\hline 23 & NONMMUT033142 & 8 & 63 & 71 \\
\hline 24 & Gm32861 & 6 & 60 & 66 \\
\hline 25 & NONMMUT051738 & 8 & 58 & 66 \\
\hline 26 & NONMMUT048637 & 8 & 56 & 64 \\
\hline 27 & NONMMUT010786 & 6 & 57 & 63 \\
\hline 28 & NONMMUT059817 & 4 & 58 & 62 \\
\hline 29 & NONMMUT050190 & 7 & 55 & 62 \\
\hline 30 & NONMMUT041236 & 8 & 54 & 62 \\
\hline 31 & 1700001L05Rik & 7 & 54 & 61 \\
\hline 32 & NONMMUT068223 & 6 & 54 & 60 \\
\hline 33 & NONMMUT064230 & 6 & 54 & 60 \\
\hline 34 & NONMMUT044605 & 6 & 53 & 59 \\
\hline 35 & NONMMUT057586 & 8 & 48 & 56 \\
\hline 36 & NONMMUT053611 & 6 & 46 & 52 \\
\hline 37 & NONMMUT036431 & 7 & 45 & 52 \\
\hline 38 & NONMMUT039492 & 6 & 43 & 49 \\
\hline 39 & NONMMUT042546 & 6 & 41 & 47 \\
\hline 40 & Gm32374 & 6 & 36 & 42 \\
\hline 41 & NONMMUT022800 & 6 & 27 & 33 \\
\hline 42 & NONMMUT045026 & 6 & 23 & 29 \\
\hline 43 & NONMMUT025285 & 6 & 11 & 17 \\
\hline
\end{tabular}

LncRNA, long non-coding RNA; miRNAs, microRNAs.

\section{Discussion}

$\mathrm{HF}$ is well recognized as a wound-healing response that occurs in liver following any type of acute or chronic injury (31-33). It is hypothesized that liver fibrosis is reversible; however, the resulting structural damage to liver lobules and vasculature leads to cirrhosis, which is irreversible (34-36). Therefore, there is an increasing requirement for diagnostic and prognostic biomarkers of HF.

Increasing evidence has demonstrated that LncRNAs are involved in numerous complex diseases by communicating with mRNAs and miRNAs, and with each other (37). Compared 

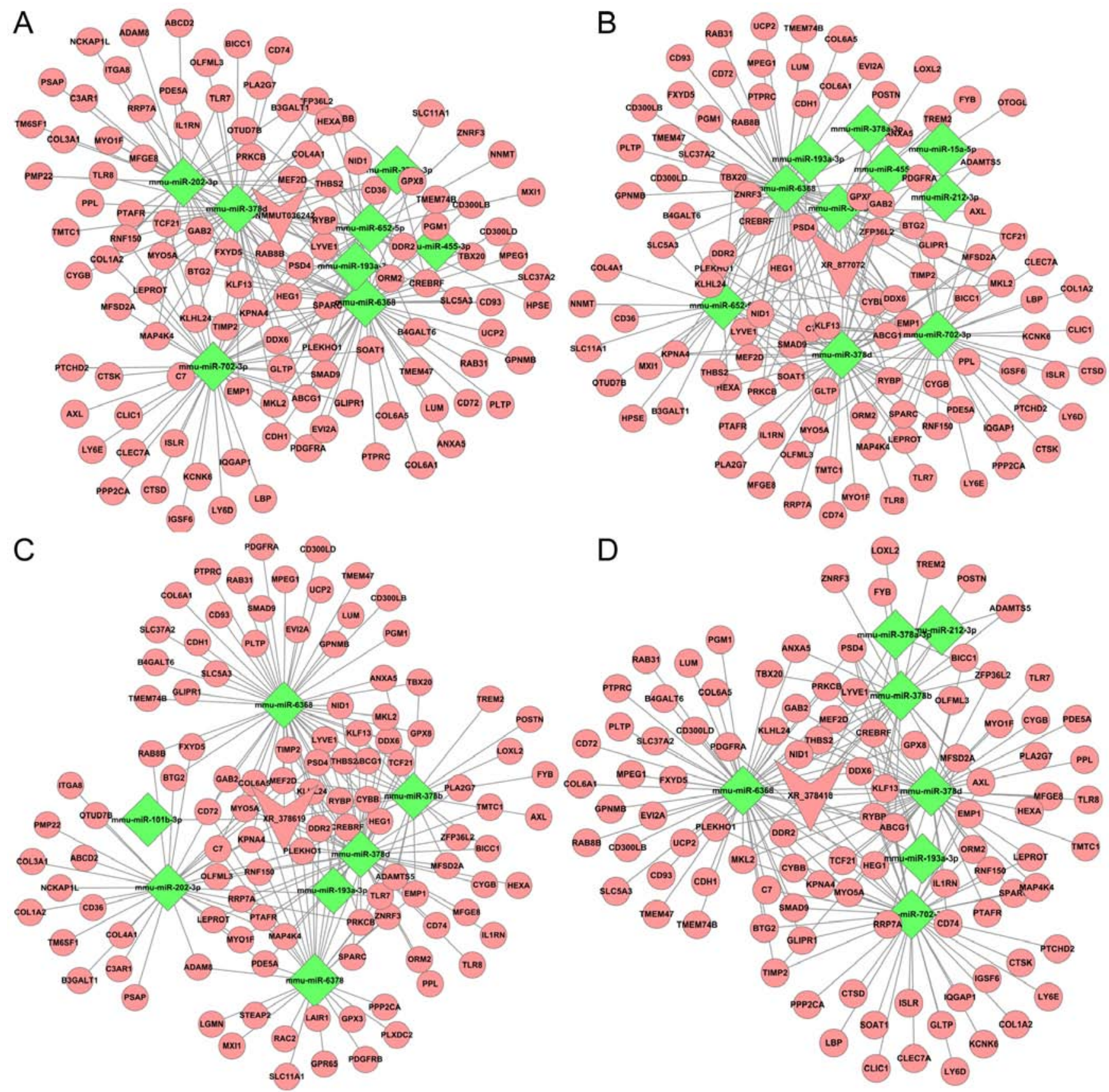

Figure 4. Key LncRNA-miRNA-mRNA sub-networks. (A) LncRNA NONMMUT036242, (B) LncRNA XR_877072, (C) LncRNA XR_378619 and (D) LncRNA XR_378418 sub-networks. The arrowheads represent LncRNAs, rhombuses represent miRNAs, circles represent mRNAs; upregulated genes are in pink, downregulated genes in green. LncRNA, long non-coding RNA; miRNAs microRNAs.

with protein-coding genes, LncRNAs have clear advantages as diagnostic and prognostic biomarkers. Exploiting interactions between LncRNAs and mRNAs/miRNAs to LncRNA functional similarity is an effective method to explore function of LncRNAs (38-40). The present study, on the basis of the ceRNA hypothesis, used interaction data generated using datasets from GEO to reconstruct a triple global network. The LncRNA-miRNA-mRNA network consisted of 220 LncRNAs, 24 miRNAs, 164 mRNAs and 1,149 interactions.

Furthermore, to investigate the functions of HF-related genes, these genes were annotated via GO and KEGG pathway analysis, and it was found that the most significant enriched terms and pathways were regulation of cytokine and collagen, and TGF- $\beta$ and TLR signaling. It is well known that activated hepatic stellate cells (HSCs) serve a role in liver fibrosis (41). Several studies have demonstrated that TGF- $\beta$ plays a critical role in HSC activation, and that the TGF- $\beta$ signaling pathway could be a potential therapeutic target for HF (42-44). This classic signaling pathway is activated by TGF- $\beta$ binding to its receptors located on the cell membrane. The downstream proteins, including Smad2 and Smad3, are activated by phosphorylation, which further promotes the transcription of genes encoding ECM components, thereby accelerating the development of liver fibrosis (45).

Previous studies have demonstrated that hub nodes, characterized by their high degree of connectivity to other 

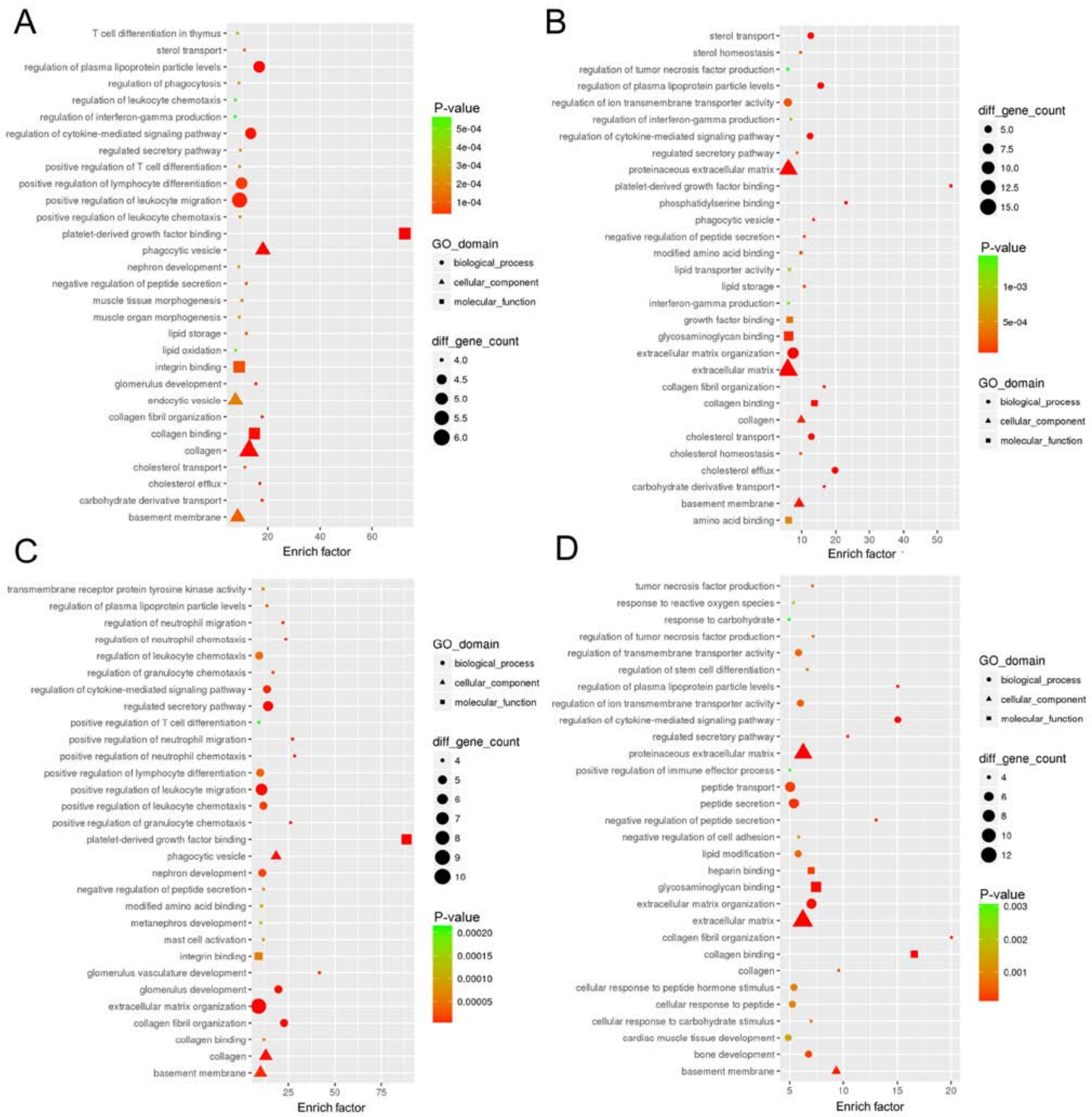

Figure 5. GO analysis of the key LncRNA-miRNA-mRNA sub-networks. The top 30 significantly enriched GO terms of the (A) LncRNA NONMMUT036242, (B) LncRNA XR_877072, (C) LncRNA XR_378619 and (D) LncRNA XR_378418-miRNA-mRNA sub-networks. Circles represent biological process, triangles represent cellular component, square represent molecular function. The different colors from green to red represent the P-value. The deeper the color, the more significant the P-value. The different sizes of the shapes represent the gene count. GO, Gene Ontology; LncRNA, long non-coding RNA; miRNAs, microRNAs.

nodes, can be used as topological properties of the network to evaluate the importance of genes $(46,47)$. In general, a LncRNA that has more edges indicates that the LncRNA is a hub, which participates in more ceRNA interactions. Thus, the LncRNA is essential in network organization and plays a critical role in a network. In the present study, according to the node degree and relationship pairs, 4 LncRNAs (NONMMUT036242, XR_877072, XR_378619 and XR_378418) were selected as the key LncRNAs, which may be used as potential novel biomarkers for the clinical diagnosis and treatment of HF.
Several LncRNAs, which are altered during HF and could affect $\mathrm{HSC}$ activation, have already been reported, including liver fibrosis-associated LncRNA1 (21), metastasis-associated lung adenocarcinoma transcript 1 (48), LncRNA-p21 (49) and maternally expressed 3 (50). However, functional characterization of the 4 key LncRNAs (NONMMUT036242, XR_877072, XR_378619 and XR_378418) is still in its infancy. In order to infer the potential functions of LncRNAs, according to the ceRNA theory, the related miRNAs and/or mRNAs, whose functions have been annotated, can be studied. While the LncRNA-miRNA-mRNA could provide a global 
A

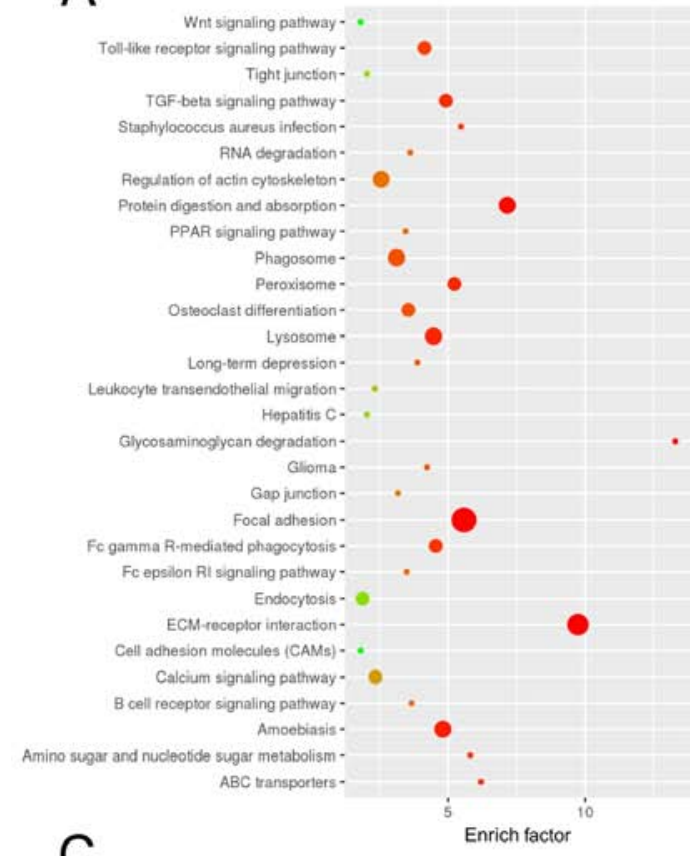

C

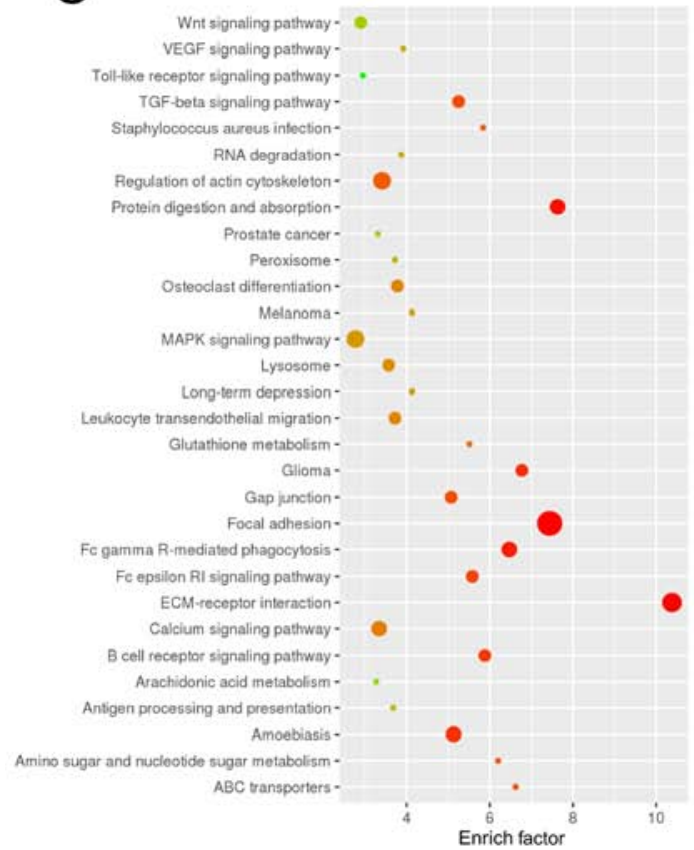

B
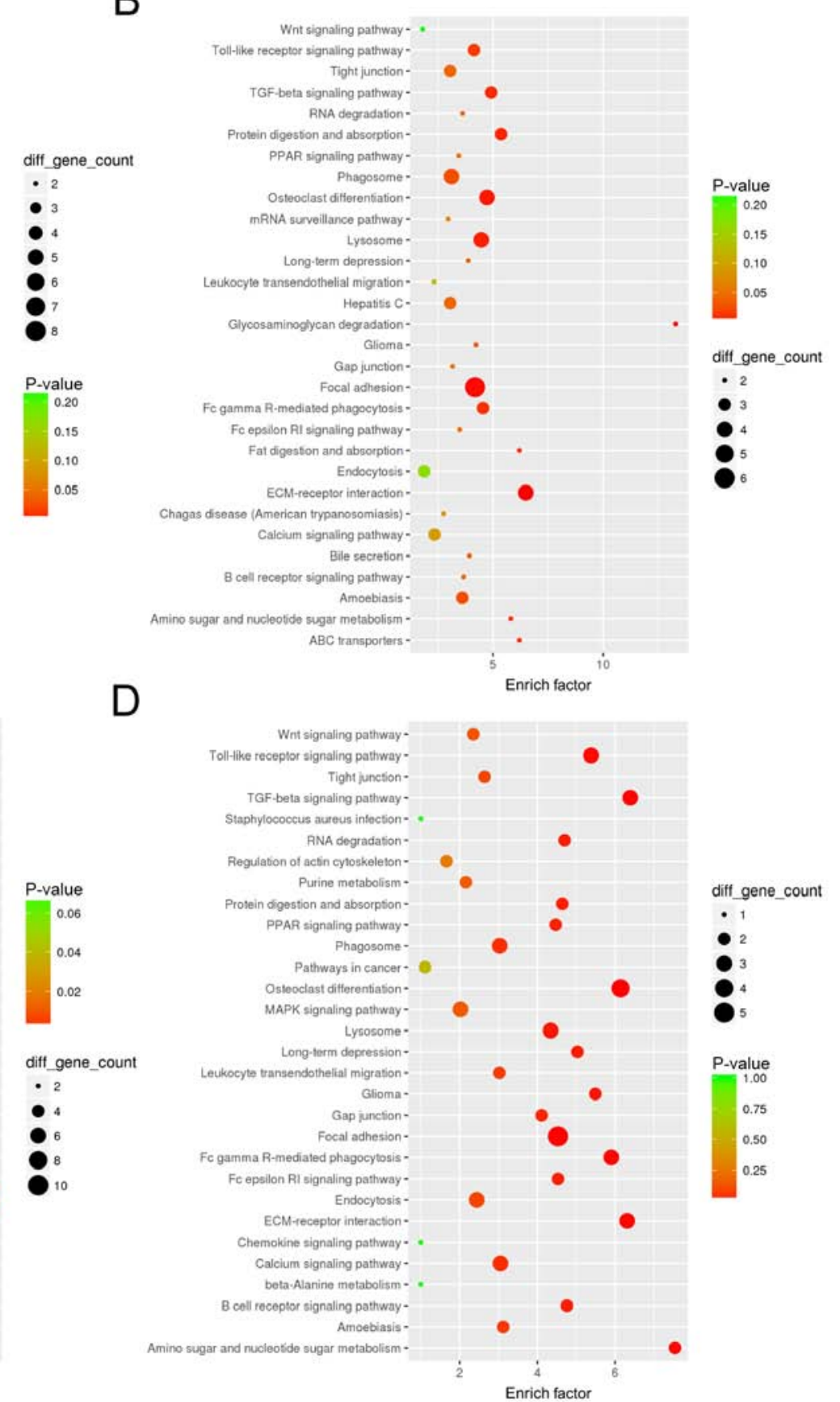

Figure 6. KEGG pathway analysis of the key LncRNA-miRNA-mRNA sub-networks. Top 30 enriched KEGG pathways of the (A) LncRNA NONMMUT036242, (B) LncRNA XR_877072, (C) LncRNA XR_378619 and (D) LncRNA XR_378418-miRNA-mRNA sub-networks. The different colors from green to red represent the P-value. The deeper the color, the more significant the P-value. The different sizes of the shapes represent the gene count number. LncRNA, long non-coding RNA; miRNAs, microRNAs; KEGG, Kyoto Encyclopedia of Genes and Genomes.

view of all possible ceRNA interactions that could be used to investigate the regulatory properties of the LncRNAs, the key LncRNA-miRNA-mRNA sub-network revealed amore detailed picture of how the key LncRNAs synergized with competing mRNAs (51). According to the key LncRNA-miRNA-mRNA sub-networks, the LncRNA XR_877072, a seldom reported LncRNA, interacted with numerous mRNAs, including CD74, discoidin domain receptor 2 and tissue inhibitor of metallopeptidases 2 , which were highly associated with HF (52-54). In addition, this LncRNA could directly interact with a number of miRNAs, including miR-378a-3p and miR-212-3p, which have been verified as important factors in the development of HF. For example, Hyun et al (55) reported that expression of miR-378a-3p declined in mice models of liver fibrosis. In activated HSCs transfected with miR-378a-3p mimic, the profibrotic gene expression of Vimentin, $\alpha$-smooth muscle actin and collagen $I \alpha 1$ decreased. In addition, the levels of Gli3, a critical target gene of the Hedgehog signaling pathway that promotes $\mathrm{HF}(56,57)$, were also downregulated $(55)$. Zhu et al (58) confirmed that overexpression of miR-212-3p can induce the expression of HSC markers, including $\alpha$-SMA, and collagens by activating the TGF- $\beta$ signaling pathway. 
In conclusion, based on the ceRNA theory, the present study constructed an HF-associated LncRNA-miRNA-mRNA network to explore the biological functions of LncRNAs during the development of $\mathrm{HF}$ at a system-wide level. Notably, 4 LncRNAs (NONMMUT036242, XR_877072, XR_378619 and XR_378418) were selected that participated in the most ceRNA interactions, and may play important roles in HF. The present study uncovered a ceRNA network that could further the understanding of the mechanisms underlying HF progression and provide novel potential markers for clinical diagnosis and targets for treatment. However, there were still certain limitations to the present study. First, the 4 key LncRNAs need to be verified in liver tissue specimens from patients with liver fibrosis via reverse transcription-quantitative PCR analysis. Second, further experiments are required to validate their effects and mechanisms in HF. Finally, the homology of the 4 key LncRNAs, which were identified based on expression in mice, needs to be validated to support their use as potential biomarkers and therapeutic targets in clinical settings.

\section{Acknowledgements}

The authors would like to thank Mr Qiang Fan (Ao Ji Bio-tech Co., Ltd., Shanghai, China) for providing help with data analysis.

\section{Funding}

The present study was supported by Leading Talents Introduction and Cultivation Plan Project of Colleges in Anhui Province (grant no. gxfxZD2016118) and the National Natural Science Foundation of China (grant no. 81973648).

\section{Availability of data and materials}

The datasets used and/or analyzed during the present study are available from the corresponding author on reasonable request.

\section{Authors' contributions}

JZ and JG made substantial contributions to the conception and design of the study. HJ, FW and NJ contributed to data acquisition, and data analysis and interpretation. HJ and JG drafter and revised the manuscript critically for important intellectual content. All authors agreed to be accountable for all aspects of the work in ensuring that questions related to the accuracy or integrity of the work are appropriately investigated and resolved. All authors read and approved the final manuscript.

\section{Ethics approval and consent to participate}

Not applicable.

\section{Patient consent for publication}

Not applicable.

\section{Competing interests}

The authors declare that they have no competing interests.

\section{References}

1. Ni MM, Wang YR, Wu WW, Xia CC, Zhang YH, Xu J, Xu T and Li J: Novel Insights on Notch signaling pathways in liver fibrosis. Eur J Pharmacol 826: 66-74, 2018.

2. Li H, Lan J, Han C, Guo K, Wang G, Hu J, Gong J, Luo X and Cao Z: Brg1 promotes liver fibrosis via activation of hepatic stellate cells. Exp Cell Res 364: 191-197, 2018.

3. Chen W, Zhang Z, Yao Z, Wang L, Zhang F, Shao J, Chen A and Zheng S: Activation of autophagy is required for Oroxylin A to alleviate carbon tetrachloride-induced liver fibrosis and hepatic stellate cell activation. Int Immunopharmacol 56: 148-155, 2018.

4. Panebianco C, Oben JA, Vinciguerra M and Pazienza V: Senescence in hepatic stellate cells as a mechanism of liver fibrosis reversal: A putative synergy between retinoic acid and PPAR-gamma signalings. Clin Exp Med 17: 269-280, 2017.

5. Tomita K, Teratani T, Suzuki T, Shimizu M, Sato H, Narimatsu K, Okada Y, Kurihara C, Irie R, Yokoyama H, et al: Free cholesterol accumulation in hepatic stellate cells: Mechanism of liver fibrosis aggravation in nonalcoholic steatohepatitis in mice. Hepatology 59: 154-169, 2014.

6. He Y, Jin L, Wang J, Yan Z, Chen T and Zhao Y: Mechanisms of fibrosis in acute liver failure. Liver Int 35: 1877-1885, 2015.

7. Jiang H, Ma R, Zou S, Wang Y, Li Z and Li W: Reconstruction and analysis of the lncRNA-miRNA-mRNA network based on competitive endogenous RNA reveal functional lncRNAs in rheumatoid arthritis. Mol Biosyst 13: 1182-1192, 2017.

8. Qu Z and Adelson DL: Evolutionary conservation and functional roles of ncRNA. Front Genet 3: 205, 2012.

9. Zheng B, Jeong S, Zhu Y, Chen L and Xia Q: miRNA and lncRNA as biomarkers in cholangiocarcinoma (CCA). Oncotarget 8: 100819-100830, 2017.

10. Huang GW, Xue YJ, Wu ZY, Xu XE, Wu JY, Cao HH, Zhu Y, He JZ, Li CQ, Li EM and Xu LY: A three-lncRNA signature predicts overall survival and disease-free survival in patients with esophageal squamous cell carcinoma. BMC Cancer 18: 147, 2018.

11. Ding L, Wang M, Sun D and Li A: TPGLDA: Novel prediction of associations between lncRNAs and diseases via lncRNA-disease-gene tripartite graph. Sci Rep 8: 1065, 2018.

12. Ye Y, Gao X and Yang N: LncRNA ZFAS1 promotes cell migration and invasion of fibroblast-like synoviocytes by suppression of miR-27a in rheumatoid arthritis. Hum Cell 31: 14-21, 2018

13. Grelet S, Link LA, Howley B, Obellianne C, Palanisamy V, Gangaraju VK, Diehl JA and Howe PH: A regulated PNUTS mRNA to lncRNA splice switch mediates EMT and tumour progression. Nat Cell Biol 19: 1105-1115, 2017.

14. Szafranski P, Dharmadhikari AV, Brosens E, Gurha P, Kolodziejska KE, Zhishuo O, Dittwald P, Majewski T, Mohan KN, Chen B, et al: Small noncoding differentially methylated copy-number variants, including lncRNA genes, cause a lethal lung developmental disorder. Genome Res 23: 23-33, 2013.

15. Li F, Huang C, Li Q and Wu X: Construction and comprehensive analysis for dysregulated long non-coding RNA (lncRNA)-associated competing endogenous RNA (ceRNA) network in gastric cancer. Med Sci Monit 24: 37-49, 2018.

16. Jiang X, Wu X, Chen F, He W, Chen X, Liu L and Tang H: The profiles and networks of miRNA, IncRNA, mRNA, and circRNA in benzo(a)pyrene-transformed bronchial epithelial cells. J Toxicol Sci 43: 281-289, 2018.

17. Xue WH, Fan ZR, Li LF, Lu JL, Ma BJ, Kan QC and Zhao J: Construction of an oesophageal cancer-specific ceRNA network based on miRNA, IncRNA, and mRNA expression data. World $\mathbf{J}$ Gastroenterol 24: 23-34, 2018.

18. Mao Y, Liu R, Zhou H, Yin S, Zhao Q, Ding X and Wang H: Transcriptome analysis of miRNA-lncRNA-mRNA interactions in the malignant transformation process of gastric cancer initiation. Cancer Gene Ther 24: 267-275, 2017.

19. Salmena L, Poliseno L, Tay Y, Kats L and Pandolfi PP: A ceRNA hypothesis: The Rosetta Stone of a hidden RNA language? Cell 146: 353-358, 2011.

20. Hyun J, Park J, Wang S, Kim J, Lee HH, Seo YS and Jung Y: MicroRNA expression profiling in $\mathrm{CCl}_{4}$-induced liver fibrosis of Mus musculus. Int J Mol Sci 17: 961, 2016.

21. Zhang K, Han X, Zhang Z, Zheng L, Hu Z, Yao Q, Cui H, Shu G, Si M, Li C, et al: The liver-enriched lnc-LFAR1 promotes liver fibrosis by activating TGF $\beta$ and Notch pathways. Nat Commun 8: $144,2017$.

22. Gao JR, Qin XJ, Jiang H, Gao YC, Guo MF and Jiang NN: Potential role of lncRNAs in contributing to pathogenesis of chronic glomerulonephritis based on microarray data. Gene 643: 46-54, 2018. 
23. Betel D, Koppal A, Agius P, Sander C and Leslie C: Comprehensive modeling of microRNA targets predicts functional non-conserved and non-canonical sites. Genome Biol 11: R90, 2010.

24. Agarwal V, Bell GW, Nam JW and Bartel DP: Predicting effective microRNA target sites in mammalian mRNAs. Elife 4, 2015.

25. Shannon P, Markiel A, Ozier O, Baliga NS, Wang JT, Ramage D, Amin N, Schwikowski B and Ideker T: Cytoscape: A software environment for integrated models of biomolecular interaction networks. Genome Res 13: 2498-2504, 2003.

26. Ashburner M, Ball CA, Blake JA, Botstein D, Butler H, Cherry JM, Davis AP, Dolinski K, Dwight SS, Eppig JT, et al: Gene ontology: Tool for the unification of biology. The Gene Ontology Consortium. Nat Genet 25: 25-29, 2000.

27. The Gene Ontology Consortium: The Gene Ontology Resource: 20 years and still GOing strong. Nucleic Acids Res 47: D330-D338, 2019.

28. Kanehisa M, Sato Y, Furumichi M, Morishima K and Tanabe M: New approach for understanding genome variations in KEGG Nucleic Acids Res 47: D590-D595, 2019.

29. Kanehisa M and Goto S: KEGG: Kyoto encyclopedia of genes and genomes. Nucleic Acids Res 28: 27-30, 2000.

30. Han JD, Bertin N, Hao T, Goldberg DS, Berriz GF, Zhang LV, Dupuy D, Walhout AJ, Cusick ME, Roth FP and Vidal M Evidence for dynamically organized modularity in the yeast protein-protein interaction network. Nature 430: 88-93, 2004.

31. Cao H, Li S, Xie R, Xu N, Qian Y, Chen H, Hu Q, Quan Y, Yu Z, Liu J and Xiang M: Exploring the mechanism of dangguiliuhuang decoction against hepatic fibrosis by network pharmacology and experimental validation. Front Pharmacol 9: 187, 2018.

32. Li P, Li Y, Zhu L, Yang Z, He J, Wang L, Shang Q, Pan H, Wang H, Ma X, et al: Targeting secreted cytokine BMP9 gates the attenuation of hepatic fibrosis. Biochim Biophys Acta Mol Basis Dis 1864: 709-720, 2018.

33. Matsuda M, Tsurusaki S, Miyata N, Saijou E, Okochi H, Miyajima A and Tanaka M: Oncostatin M causes liver fibrosis by regulating cooperation between hepatic stellate cells and macrophages in mice. Hepatology 67: 296-312, 2018.

34. Chi C, Liu XY, Hou F, Yu XZ, Li CY, Cui LJ, Liu RX and Yin CH: Herbal compound 861 prevents hepatic fibrosis by inhibiting the TGF- $\beta 1 / \mathrm{Smad} / \mathrm{SnoN}$ pathway in bile duct-ligated rats. BMC Complement Altern Med 18: 52, 2018.

35. Omar R, Yang J, Liu H, Davies NM and Gong Y: Hepatic stellate cells in liver fibrosis and siRNA-based therapy. Rev Physiol Biochem Pharmacol 172: 1-37, 2016.

36. Pascher A, Nebrig M and Neuhaus P: Irreversible liver failure: Treatment by transplantation: Part 3 of a series on liver cirrhosis. Dtsch Arztebl Int 110: 167-173, 2013.

37. Cheng L, Shi H, Wang Z, Hu Y, Yang H, Zhou C, Sun J and Zhou M: IntNetLncSim: An integrative network analysis method to infer human lncRNA functional similarity. Oncotarget 7 : 47864-47874, 2016.

38. Zhao Y, Wang H, Wu C, Yan M, Wu H, Wang J, Yang X and Shao Q: Construction and investigation of lncRNA-associated ceRNA regulatory network in papillary thyroid cancer. Oncol Rep 39: 1197-1206, 2018.

39. Zhou M, Wang X, Shi H, Cheng L, Wang Z, Zhao H, Yang L and Sun J: Characterization of long non-coding RNA-associated ceRNA network to reveal potential prognostic lncRNA biomarkers in human ovarian cancer. Oncotarget 7: 12598-12611, 2016.

40. Xu JH, Chang WH, Fu HW, Yuan T and Chen P: The mRNA, miRNA and lncRNA networks in hepatocellular carcinoma: An integrative transcriptomic analysis from Gene Expression Omnibus. Mol Med Rep 17: 6472-6482, 2018.

41. Wei S, Wang Q, Zhou H, Qiu J, Li C, Shi C, Zhou S, Liu R and Lu L: miR-455-3p alleviates hepatic stellate cell activation and liver fibrosis by suppressing HSF1 expression. Mol Ther Nucleic Acids 16: 758-769, 2019

42. Chen N, Geng Q, Zheng J, He S, Huo X and Sun X: Suppression of the TGF- $\beta /$ Smad signaling pathway and inhibition of hepatic stellate cell proliferation play a role in the hepatoprotective effects of curcumin against alcohol-induced hepatic fibrosis. Int J Mol Med 34: 1110-1116, 2014.
43. Wang K, Tang Y, Yan F, Zhu J and Li J: Potent inhibition of TGF- $\beta$ signaling pathway regulator Abl: Potential therapeutics for hepatic fibrosis. J Recept Signal Transduct Res 35: 410-419, 2015.

44. Xu A, Li Y, Zhao W, Hou F, Li X, Sun L, Chen W, Yang A, Wu S, Zhang B, et al: PHP14 regulates hepatic stellate cells migration in liver fibrosis via mediating TGF- $\beta 1$ signaling to PI3K $\gamma / \mathrm{AKT} / \mathrm{Racl}$ pathway. J Mol Med (Berl) 96: 119-133, 2018.

45. Zhou L, Dong X, Wang L, Shan L, Li T, Xu W, Ding Y, Lai M, Lin X, Dai M, et al: Casticin attenuates liver fibrosis and hepatic stellate cell activation by blocking TGF- $\beta /$ Smad signaling pathway. Oncotarget 8: 56267-56280, 2017.

46. Zhang Y, Xu Y, Feng L, Li F, Sun Z, Wu T, Shi X, Li J and Li X: Comprehensive characterization of lncRNA-mRNA related ceRNA network across 12 major cancers. Oncotarget 7: 64148-64167, 2016.

47. Zhou M, Diao Z, Yue X, Chen Y, Zhao H, Cheng L and Sun J: Construction and analysis of dysregulated lncRNA-associated ceRNA network identified novel lncRNA biomarkers for early diagnosis of human pancreatic cancer. Oncotarget 7: 56383-56394, 2016.

48. Leti F, Legendre C, Still CD, Chu X, Petrick A, Gerhard GS and DiStefano JK: Altered expression of MALAT1 lncRNA in nonalcoholic steatohepatitis fibrosis regulates CXCL5 in hepatic stellate cells. Transl Res 190: 25.e21-39.e21, 2017.

49. Yu F, Lu Z, Chen B, Dong P and Zheng J: Identification of a novel lincRNA-p21-miR-181b-PTEN signaling cascade in liver fibrosis. Mediators Inflamm 2016: 9856538, 2016.

50. Chen MJ, Wang XG, Sun ZX and Liu XC: Diagnostic value of LncRNA-MEG3 as a serum biomarker in patients with hepatitis B complicated with liver fibrosis. Eur Rev Med Pharmacol Sci 23: 4360-4367, 2019.

51. Cao Y, Wang P, Ning S, Xiao W, Xiao B and Li X: Identification of prognostic biomarkers in glioblastoma using a long non-coding RNA-mediated, competitive endogenous RNA network. Oncotarget 7: 41737-41747, 2016.

52. Heinrichs D, Knauel M, Offermanns C, Berres ML, Nellen A, Leng L, Schmitz P, Bucala R, Trautwein C, Weber C, et al: Macrophage migration inhibitory factor (MIF) exerts antifibrotic effects in experimental liver fibrosis via CD74. Proc Natl Acad Sci USA 108: 17444-17449, 2011.

53. Zhang XH, Yan M, Liu L, Wu TJ, Ma LL and Wang LX: Expression of discoidin domain receptors (DDR2) in alcoholic liver fibrosis in rats. Arch Med Res 41: 586-592, 2010.

54. Yang J, Zheng J, Wu L, Shi M, Zhang H, Wang X, Xia N, Wang D, Liu X, Yao L, et al: NDRG2 ameliorates hepatic fibrosis by inhibiting the TGF- $\beta 1 /$ Smad pathway and altering the MMP2/TIMP2 ratio in rats. PLoS One 6: e27710, 2011.

55. Hyun J, Wang S, Kim J, Rao KM, Park SY, Chung I, Ha CS, Kim SW, Yun YH and Jung Y: MicroRNA-378 limits activation of hepatic stellate cells and liver fibrosis by suppressing Gli3 expression. Nat Commun 7: 10993, 2016.

56. Zhang F, Hao M, Jin H, Yao Z, Lian N, Wu L, Shao J, Chen A and Zheng S: Canonical hedgehog signalling regulates hepatic stellate cell-mediated angiogenesis in liver fibrosis. Br J Pharmacol 174: 409-423, 2017.

57. Chung SI, Moon H, Ju HL, Cho KJ, Kim DY, Han KH, Eun JW, Nam SW, Ribback S, Dombrowski F, et al: Hepatic expression of Sonic Hedgehog induces liver fibrosis and promotes hepatocarcinogenesis in a transgenic mouse model. J Hepatol 64: 618-627, 2016.

58. Zhu J, Zhang Z, Zhang Y, Li W, Zheng W, Yu J, Wang B, Chen L, Zhuo Q, Chen L, et al: MicroRNA-212 activates hepatic stellate cells and promotes liver fibrosis via targeting SMAD7. Biochem Biophys Res Commun 496: 176-183, 2018.

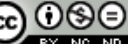

This work is licensed under a Creative Commons Attribution-NonCommercial-NoDerivatives 4.0 International (CC BY-NC-ND 4.0) License. 